\title{
Dietary Behaviours Among Han, Tujia and Miao Primary School Students: A Cross-Sectional Survey in Chongqing, China
}

This article was published in the following Dove Press journal: Risk Management and Healthcare Policy

\author{
Zhengjie Cai ${ }^{1-3, *}$ \\ Jinli Xian ${ }^{1-3, *}$ \\ Xianglong $\mathrm{Xu}\left(\mathbb{I D}^{4-6}\right.$ \\ Ziwei Zhang ${ }^{7}$ \\ Camila Araujo ${ }^{8}$ \\ Manoj Sharma (iD 9 \\ Yong Zhao (D) ${ }^{1-3,10}$
}

'School of Public Health and Management, Chongqing Medical University, Chongqing, People's Republic of China; ${ }^{2}$ Research Center for Medicine and Social Development, Chongqing Medical University, Chongqing, People's Republic of China; ${ }^{3}$ The Innovation Center for Social Risk Governance in Health, Chongqing Medical University, Chongqing, People's Republic of China; ${ }^{4}$ Central Clinical School, Faculty of Medicine, Nursing and Health Sciences, Monash University, Melbourne, VIC, Australia; ${ }^{5}$ Melbourne Sexual Health Centre, Alfred Health, Melbourne, VIC, Australia; ${ }^{6}$ China-Australia Joint Research Center for Infectious Diseases, School of Public Health, Xi'an Jiaotong University Health, Science Center, Xi'an, Shanxi, People's Republic of China; ${ }^{7}$ The Second Clinical College, Chongqing Medical University, Chongqing, People's Republic of China; ${ }^{8}$ Department of Physiology and Pharmacology, Loma Linda University School of Medicine, Loma Linda, CA, USA; ${ }^{9}$ Environmental and Occupational Health, School of Public Health, University of Nevada, Las Vegas, NV, USA; ${ }^{10}$ Chongqing Key Laboratory of Child Nutrition and Health, Children's Hospital of Chongqing Medical University, Chongqing, People's Republic of China

*These authors contributed equally to this work

Correspondence: Yong Zhao

Email zhaoyong@cqmu.edu.cn
Background and Purpose: Exploring the differences of children's dietary behaviours from different nationalities could provide a reference for targeted nutritional interventions. This study aims to assess the dietary behaviours among Han, Tujia and Miao primary school students and the differences among the ethnic groups.

Materials and Methods: This cross-sectional survey included 556 primary school students (249 Han, 76 Tujia and 231 Miao students) aged 6-13 years from the representative minority areas of Chongqing, China. Data were collected with a self-administered or one-on-one interview-based questionnaire encompassing questions on sociodemographics, eating habits and dietary intake. Eating habits and dietary intake were categorized into health-protective and health-risk dietary behaviours. $\chi^{2}$ tests were conducted to examine the differences in eating habits and dietary intake among the three ethnic groups. Forward stepwise logistic regression models were performed to check the factors affecting dietary intake.

Results: Fewer than half of the students had health-protective dietary behaviours in terms of rice, fruit, fish, eggs and milk intake. Compared with Han students, Tujia students were more likely to meet the recommended intake of vegetables ( $\mathrm{OR}=2.13,95 \%$ CI $[1.22-3.69])$, less likely to meet the recommended intake of meat ( $\mathrm{OR}=0.58,95 \% \mathrm{CI}[0.35-0.94])$ and fish (OR $=0.55,95 \% \mathrm{CI}[0.32-0.94])$; Miao students were more likely to meet the recommended intake of vegetables $(\mathrm{OR}=1.73,95 \% \mathrm{CI}[1.20-2.51])$, fruits $(\mathrm{OR}=1.62,95 \%$ CI $[1.16-2.28])$ and water $(\mathrm{OR}=1.59,95 \% \mathrm{CI}[1.12-2.26])$ than those of Han students.

Conclusion: The low proportion of health-protective dietary behaviours among primary school students from Han, Tujia and Miao ethnicities was found in this study. There were differences in dietary behaviours among the three ethnic groups. This study could provide a reference for implementing national dietary guidelines in consideration of socioeconomic status, ways of preparing and consuming foods, traditional foods and customs of different ethnic groups.

Keywords: dietary behaviours, dietary guidelines, minority nationalities, primary school students

\section{Introduction}

Healthy dietary behaviours play a vital role in promoting optimal growth and development of children, ${ }^{1}$ moreover, dietary behaviours established during childhood are likely to persist into adulthood. ${ }^{2,3}$ Considering these factors, promoting healthy eating of children should be a priority for public health research. ${ }^{4}$

China is a multi-ethnic country with 56 distinct ethnicities. Han ethnicity accounts for the majority, the other 55 ethnicities are considered as ethnic minorities, who make up nearly $8 \%$ of the entire Chinese population. ${ }^{5}$ Previous studies 
have reported the racial and ethnic disparities in the prevalence of chronic diseases (such as cardiovascular disease and diabetes) in China. ${ }^{6,7}$ Some scholars proposed that over-nutrition of Han nationality and under-nutrition of ethnic minorities among children and adolescents in China need to be taken seriously. ${ }^{8}$ As suggested by previous research and the WHO's report, dietary factors play a crucial role in the development of chronic diseases and obesity. ${ }^{9,10}$ Different socioeconomic status, ways of preparing and consuming foods, traditional foods and customs of different ethnic groups in China may cause the differences in their dietary behaviours. ${ }^{11,12}$ Fengying's study with the objective of investigating the status and characteristic of dietary intake of 12 minority nationalities in China showed that rice was the staple food for Tujia and Miao ethnic minorities, low intake of milk and eggs was observed in the two ethnic minorities; specifically, high intake of soybean products and cooking oil was characterized for Tujia population, low intake of fruits and aquatic products and high intake of cooking salt were characterized for Miao population. ${ }^{11}$ A comparative study of healthrelated behaviours in Han and minority children in China showed that the proportion of Han children who preferred vegetables, fruits and soft drink was higher than that of ethnic minorities in China. ${ }^{13}$ It is of great significance to analyze ethnic differences in dietary behaviours, which is helpful to formulate targeted policies and implement interventions for improving the dietary behaviours of these specific ethnic groups.

Dietary guidelines were formulated to develop a set of core appropriate dietary messages for different age groups based on current food and nutrient requirements, and emerging nutritional concerns in different countries, in the hope of promoting overall nutritional health and preventing the development of nutrition-related diseases or conditions. ${ }^{14-17}$ During the period of nutritional transitions from traditional diets to westernized diets in China, Chinese Dietary Guideline (CDG) $2016^{16}$ was the latest version produced by Chinese government and it provided six core dietary recommendations for healthy people over 2 years of age in the general population: (1) eat a variety of foods, mainly cereals; (2) balance eating and exercise to maintain a healthy body weight; (3) consume plenty of vegetables, fruits, milk, and soybeans; (4) consume appropriate amounts of fish, poultry, eggs, and lean meat; (5) reduce cooking oil and salt intake, and limit sugar and alcohol intake; (6) eliminate waste and develop a new ethos of diet civilization. Also, the amounts of daily cereals, vegetables, fruits, eggs, aquatic product, meat, soybean, nuts, milk and milk products, salt, and oil intake were recommended in the CDG. ${ }^{16}$

Overall diet quality can be assessed by dietary index, which was formulated based on the CDG, and there was an association between overall diet quality and overweight/obesity among Chinese children and adolescents. ${ }^{18}$ Moreover, Xianwen et al demonstrated that primary school students with healthy dietary pattern, characterized by high intake of milk/yogurt, fruits, eggs and vegetables and low intake of meat such as pork, poultry, organ meat, had a lower risk of obesity and abdominal obesity. ${ }^{19}$ However, previous studies have shown that unhealthy dietary behaviours among children are becoming increasingly prevalent in different countries. ${ }^{14,20,21}$ In the United States, a study found that children were not meeting the recommended intake of vegetables, beans, and whole grains as established by the American dietary guidelines. ${ }^{20}$ Additionally, in Switzerland, it was demonstrated that children did not meet the recommendations of the Swiss Society for Nutrition dietary guidelines, by consuming too many sweets, snacks, and soft drinks, and too few fruits and vegetables. ${ }^{14}$ Simultaneously, in China, a previous study showed that among the 439 primary and secondary students aged 9-20 years (Han students accounting for $91.6 \%$ ), only $1.82 \%$ students' average daily intake of meat, eggs, dairy products, soybean products, fresh vegetables and fruits met the CDG recommended intake, the dietary pattern was extremely unbalanced. ${ }^{21}$

To the best of our knowledge, no study has assessed the health-protective and health-risk dietary behaviours based on the CDG 2016 among children from different ethnic groups in China, this topic is more focused and deserves further study. Chongqing is located in the southwest of China, southwestern region has the largest number of ethnic minorities in China. ${ }^{22}$ The largest minority population in Chongqing was Tujia ethnic minority, followed by Miao ethnic minority. ${ }^{5}$ Therefore, the objectives of the study are: (1) to assess the dietary behaviours among primary school students from Han, Tujia and Miao nationalities in Chongqing, China; (2) to explore the differences of dietary behaviours among different ethnic groups. This study could provide a reference for the implementation of national dietary guidelines in consideration of socioeconomic status, ways of preparing and consuming foods, traditional foods and customs of different ethnic groups. 


\section{Materials and Methods}

Study Design and Participants

A cross-sectional survey on dietary behaviours was conducted among 556 primary school students aged 6-13 years in grades $1-6$ (48.4\% boys) from the representative minority areas of Chongqing, China. Han nationality constituted the main population, Miao and Tujia nationalities were the main minorities in Chongqing. ${ }^{5}$ Wulong District, Qianjiang District, Pengshui Tujia and Miao Autonomous County were randomly selected as the representative minority areas in Chongqing. One primary school was randomly selected from each of selected districts, respectively, special primary schools for the disabled children or private primary schools were excluded. Students from selected schools were invited to participate in this study. Inclusion criteria of the participants were: (1) primary school students in grades 1-6; (2) they had ability to understand the information in the questionnaire; (3) they have lived in the local area for longer than 1 year. Students who had a history of major diseases, chronic health condition, or mental trauma were excluded.

A previous study investigated the intake of meat, eggs, dairy products, soybean products, vegetables and fruits among primary and secondary students, the proportion of students meeting the recommended intake of dairy products accounted for the lowest, which is $18.91 \%{ }^{21}$ According to the formula of sample size calculation:

$$
N=\left(Z_{\alpha}^{2} \times p \times q\right) / d^{2}
$$

We set $p=0.1891, q=1-p=0.8109$, and margin of error $d=0.20 \times p=0.0378, z_{a}=1.96$, the calculated sample size was 412 . In the survey, the actual total sample size included 556 individuals.

The students were invited to fill in the anonymous questionnaire before class. Investigators briefly explained the research to the students, and they were informed that answering the questionnaire was completely voluntary, and the collected information would be treated confidentially. Considering the respondents are primary school students, their inability to read or understand this questionnaire should be considered. The students in grades 3-6 filled in the questionnaire by themselves, investigators were arranged on the spot to explain the questions that students did not understand in the questionnaire, and the data of primary school students in grades 1-2 were collected by the method of one-on-one interview-based questionnaire.

\section{Data Collection}

Data were collected by using a self-administered or one-on -one interview-based questionnaire, which included sociodemographic characteristics, eating habits and dietary intake. Sociodemographic characteristics included age, gender (male/female), residence (urban/rural), the lack of siblings (yes/no). Nationality was categorized into Han, Tujia, or Miao nationality. Grade was divided into 1st to 6th grade. Grades 1-3 were considered as the low grade, grades 4-6 were considered as the senior grade. ${ }^{23}$ Previous studies showed that there was an association between dietary intake and academic grades in primary school children, ${ }^{24,25}$ thus, grades ranking was selected as a factor that may affect dietary behaviours. Grades ranking in class was divided into four groups: the top $25 \%, 25-$ $50 \%, 50-75 \%$ and the last $25 \% .{ }^{26}$ Questions on eating habits and dietary intake were adapted from the CDG $2016 .^{16}$ Ten questions of eating habits were set as follows: (1) paying attention to eating less refined grain (yes/no); (2) having a typically light diet usually (yes/no); (3) eating sweet food usually (yes/no); (4) eating fried food usually (yes/no); (5) paying attention to eating less salt, soy sauce and other seasonings (yes/no); (6) paying attention to balancing animal and plant foods (yes/no); (7) eating potato foods (such as sweet potato, yams, taro) usually (yes/no); (8) eating too much at a meal usually (yes/no); (9) eating soybean products usually (yes/no); (10) eating eggs without yolk usually (yes/no). Respondents who answered 'yes' for questions (1), (2), (5), (6), (7), (9), and "no" for questions (3), (4), (8), (10) were classified as having health-protective dietary behaviours, respondents who answered the other option was classified as having health-risk dietary behaviours. Eight items of dietary intake were designed. For each food item, participants were asked about the quantity or frequency of food intake. In this part, investigators displayed and explained the food model of CDG 2016 on-site to help students answer the questions of dietary intake. (1) daily rice (one bowl and less, two bowls, three bowls, four bowls, five bowls, six bowls and above). (2) daily vegetables (less than $300 \mathrm{~g}$, 300-400 g, 400-500 g). (3) daily fruits (less than $200 \mathrm{~g}$, 200-350 g, 350-500 g, more than $500 \mathrm{~g}$ ). (4) eggs (never, one or two a week, three or four a week, one a day, two or three a day). (5) daily milk (never, less than $250 \mathrm{ml}, 250$ $\mathrm{ml}$, more than $250 \mathrm{ml}$ ). (6) daily water (less than $800 \mathrm{ml}$, 800-1200 ml, 1200-1400 ml, more than $1400 \mathrm{ml}$ ). (7) meat (once or twice a week or less, three or four times 
a week, once a day, twice or three times a day). (8) fish (never, once or twice a week, three or four times a week, once a day, twice or three times a day). Respondents who answered eating "three or four or five bowls" of daily rice, "300-400 g" or " $400-500$ g" of daily vegetables, "200-350 g" of daily fruits, "one a day" for egg intake, "250 ml" of daily milk, and " $800-1200 \mathrm{ml}$ " or "1200-1400 ml" of daily water, "once a day or twice a day or three times a day" of meat and fish, were marked as "yes" and considered as having health-protective dietary behaviours, respondents who answered the other options were marked as "no" and classified as having health-risk dietary behaviours. The questionnaire was reviewed by a panel of experts in the field of dietary behaviour or student nutrition, and was piloted for readability and comprehension, with appropriate adjustments before administration.

\section{Data Analysis}

All statistical analyses were performed by using Stata software (Version 12, StataCorp, College Station, TX, USA). Invalid or missing data were excluded, all data were doublechecked. Age was described using mean and standard deviation, the other sociodemographic characteristics were described using frequency and percentage. Eating habits and dietary intake were classified into two categories: health-protective and health-risk dietary behaviours, and were expressed as frequency and percentage. An analysis of variance (ANOVA) test was conducted to examine age difference among Han, Tujia and Miao primary school students. $\chi^{2}$ tests were conducted to examine the differences in sociodemographic characteristics (except for age), eating habits and dietary intake among the three ethnic groups. Forward stepwise logistic regression models were performed to check the factors affecting dietary intakes. Dietary intakes were chosen as dependent variables, sociodemographic characteristics were chosen as independent variables. We hypothesize that there are differences in dietary intakes among the three ethnic groups. Statistical significance was considered if $\mathrm{p}<0.05$ (two-sided).

\section{Quality Control}

Questions on eating habits and dietary intake were adapted from the CDG 2016, ${ }^{16}$ several previous studies provided references for the setting of sociodemographic characteristics and the options setting of questions in the questionnaire. $^{24,26-28}$ The questionnaire was repeatedly revised through expert interviews and the pilot survey.
All the investigators of this study were recruited through strictly interviews, they received unified training and were required to have a good understanding of the methods and objectives of this study, as well as rich experience in dealing with potentially sensitive issues. Moreover, the investigators have a good grasp of the food model of CDG 2016. In order to make students better understand and answer the questions in the questionnaire, investigators displayed and explained the food model to the participants, besides, the one-on-one interview-based questionnaire was used among students in grades 1-2.

\section{Ethical Aspects}

This study was approved by the Ethics Committee of Chongqing Medical University (record number 2,016,001). The legal guardians of the children knew about the research, school teachers released the message of inviting students to participate in the survey to the parents through the QQ (one of the leading social networks in China), and legal guardians expressed their consents by clicking "yes" (one of the interactive function of QQ). Then, the written informed consent for processing personal data was obtained from school teachers and verbal informed consent was obtained from students.

\section{Results}

\section{Sociodemographic Characteristics of Participants}

The sociodemographic characteristics of the participants are presented in Table 1. The average age was 10.1 years old, of which $51.6 \%$ were girls. Among them, $73.6 \%$ lived in the urban areas, and $78.6 \%$ had siblings. Approximately $60 \%$ of the students were from senior grade, and $75.9 \%$ ranked the top $50 \%$ in terms of their grades in class.

\section{Eating Habits Among Han, Tujia and Miao Primary School Students}

Results of eating habits among Han, Tujia and Miao primary school students are shown in Table 2. The differences were significant among the three ethnic groups regarding having a light diet usually, eating sweet foods usually and eating fried foods usually $(\mathrm{p}<0.05)$.

\section{Dietary Intake Among Han, Tujia and Miao Primary School Students}

The results of dietary intake among Han, Tujia and Miao primary school students are shown in Table 3 . The overall 
Table I Sociodemographic Characteristics of Han, Tujia and Miao Primary School Students in Chongqing, China

\begin{tabular}{|c|c|c|c|c|c|}
\hline Characteristics & $\begin{array}{l}\text { Total Populations } \\
(\mathrm{N}=556)\end{array}$ & $\begin{array}{l}\text { Han Nationality } \\
(N=249)\end{array}$ & $\begin{array}{l}\text { Tujia Nationality } \\
(\mathbf{N}=76)\end{array}$ & $\begin{array}{l}\text { Miao Nationality } \\
(N=23 I)\end{array}$ & $P$ value \\
\hline Age (mean, SD) & I0.I (I.4) & $10.0(1.5)$ & I0.I (I.I) & $10.2(1.3)$ & 0.21 \\
\hline \multicolumn{6}{|l|}{ Gender (n, \%) } \\
\hline Boys & $269(48.4)$ & II 4 (45.8) & $46(60.5)$ & $109(47.2)$ & 0.07 \\
\hline Girls & $287(51.6)$ & $135(54.2)$ & $30(39.5)$ & $122(52.8)$ & \\
\hline \multicolumn{6}{|l|}{ Residence (n, \%) } \\
\hline Urban & $409(73.6)$ & 194 (77.9) & $45(59.2)$ & $170(73.6)$ & $0.005 * *$ \\
\hline Rural & I 47 (26.4) & $55(22.1)$ & $31(40.8)$ & $61(26.4)$ & \\
\hline \multicolumn{6}{|l|}{ Lack of siblings (n, \%) } \\
\hline Yes & $119(21.4)$ & $63(25.3)$ & $12(15.8)$ & $44(19.0)$ & 0.11 \\
\hline No & $437(78.6)$ & I86 (74.7) & $64(84.2)$ & $187(8 \mid .0)$ & \\
\hline \multicolumn{6}{|l|}{ Grade $(\mathrm{n}, \%)$} \\
\hline 1 & $49(8.8)$ & $26(10.4)$ & $6(7.9)$ & $17(7.4)$ & $0.000 * *$ \\
\hline 2 & $37(6.7)$ & 29 (11.7) & I (I.3) & $7(3.0)$ & \\
\hline 3 & 133 (23.9) & 69 (27.7) & $13(17.1)$ & $5 I(22.1)$ & \\
\hline 4 & $116(20.9)$ & $33(13.3)$ & $36(47.4)$ & $47(20.4)$ & \\
\hline 5 & $157(28.2)$ & $42(16.9)$ & I8 (23.7) & $97(42.0)$ & \\
\hline 6 & $64(11.5)$ & $50(20.1)$ & $2(2.6)$ & $12(5.2)$ & \\
\hline \multicolumn{6}{|l|}{ Academic performance in class ( $\mathrm{n}, \%)$} \\
\hline Top $25 \%$ & $130(23.4)$ & $65(26.1)$ & $14(18.4)$ & $51(22.1)$ & 0.81 \\
\hline $25-50 \%$ & $292(52.5)$ & $124(49.8)$ & $43(56.6)$ & $125(54.1)$ & \\
\hline $50-75 \%$ & III (20.0) & $49(19.7)$ & $15(19.7)$ & $47(20.3)$ & \\
\hline Last $25 \%$ & $23(4.1)$ & II (4.4) & $4(5.3)$ & $8(3.5)$ & \\
\hline
\end{tabular}

Note: $*^{*} p<0.01$.

percentage of health-protective dietary behaviours in terms of rice, vegetables, fruit, meat, fish, eggs, milk and water intake was $23.4 \%, 57.0 \%, 35.8 \%, 64.6 \%, 41.0 \%, 27.5 \%$, $34.7 \%, 53.6 \%$, respectively. Differences were significant among the three ethnic groups regarding vegetables, water and fish intake $(\mathrm{p}<0.05)$. In addition, more specific results of dietary intake are shown in Supplementary Table 1.

\section{Stepwise Logistic Regression for Identifying the Factors Affecting Dietary Intake}

The results of the forward stepwise logistic regression analysis are shown in Figure 1. Primary school students from Tujia nationality were more likely to meet the recommended intake of vegetables than those from Han nationality ( $\mathrm{OR}=$ $2.13,95 \% \mathrm{CI}$ [1.22-3.69]), however, they were less likely to meet the recommended intake of meat $(\mathrm{OR}=0.58,95 \% \mathrm{CI}$ [0.35-0.94]) and fish (OR $=0.55,95 \%$ CI [0.32-0.94]) compared with Han nationality. Primary school students from Miao nationality were more likely to meet the recommended intake of vegetables $(\mathrm{OR}=1.73,95 \% \mathrm{CI}$ [1.20-2.51]), fruits $(\mathrm{OR}=1.62,95 \% \mathrm{CI}[1.16-2.28])$ and water $(\mathrm{OR}=1.59,95 \% \mathrm{CI}[1.12-2.26])$ than those from Han nationality. Also, gender, grades ranking, grade, the lack of siblings, residence and age were the influencing factors of rice, vegetables, meat, fish, eggs, milk and water intake. Students whose grades ranking were between $50-75 \%$ were less likely to meet the recommended intake of rice $(\mathrm{OR}=$ $0.55,95 \%$ CI $[0.32-0.96])$, eggs ( $\mathrm{OR}=0.59,95 \%$ CI [0.$36-0.99])$ and milk (OR $=0.57,95 \%$ CI [0.36-0.92]) compared with those in the top $25 \%$.

\section{Discussion}

This study demonstrated that the low proportion of health-protective dietary behaviours among primary school students from Han, Tujia and Miao ethnic groups was found in this study. Han nationality constituted the main population in China, Miao and Tujia nationalities were the main minorities in the surveyed area. This study found the differences in dietary behaviours 
Table 2 Eating Habits Among Han, Tujia and Miao Primary School Students in Chongqing, China

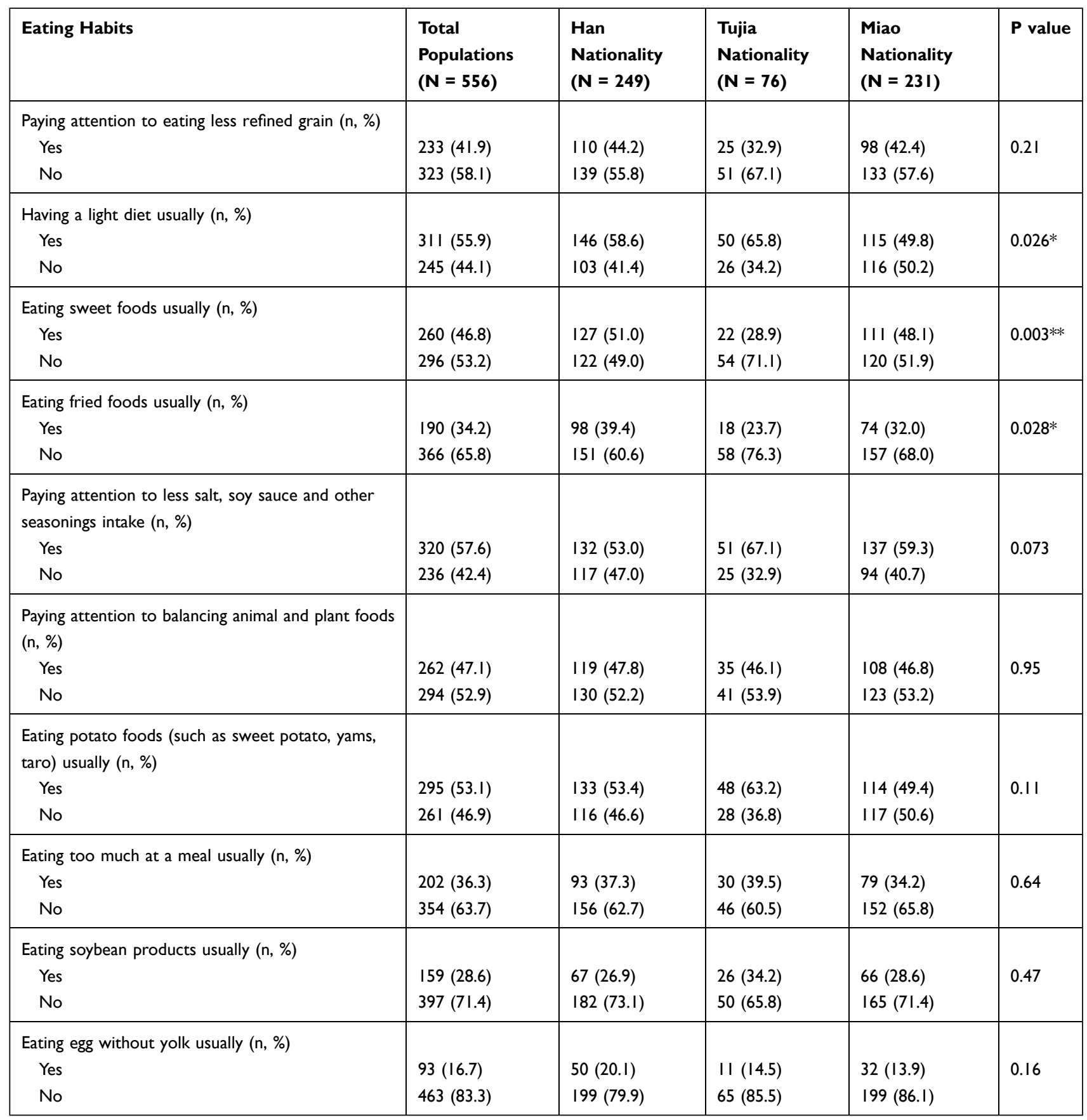

Note: ${ }^{*} p<0.05,{ }^{*} *_{p}<0.01$.

among Han, Tujia and Miao ethnic groups. Understanding the differences in dietary behaviours across ethnic groups could contribute to formulating targeted policies and interventions for improving the dietary behaviours in these specific ethnic groups in consideration of their socioeconomic status, ways of preparing and consuming foods, traditional foods and customs.
In this study, the overall low proportion of meeting the recommended intake of rice, fruit, fish, eggs, and milk intake was observed among the three nationalities, indicating that the dietary pattern of primary school students in the minority areas of Chongqing is not reasonable. Milk, eggs and fish, as the source of high-quality protein, are critical to the growth and development of primary school students who are going through puberty. The "China School Milk 
Table 3 Dietary Intake Among Han, Tujia and Miao Primary School Students in Chongqing, China

\begin{tabular}{|c|c|c|c|c|c|}
\hline Dietary Intake & $\begin{array}{l}\text { Total Populations } \\
(\mathrm{N}=556)\end{array}$ & $\begin{array}{l}\text { Han Nationality } \\
(\mathrm{N}=\mathbf{2 4 9})\end{array}$ & $\begin{array}{l}\text { Tujia Nationality } \\
(\mathrm{N}=76)\end{array}$ & $\begin{array}{l}\text { Miao Nationality } \\
(\mathrm{N}=23 \mathrm{I})\end{array}$ & $P$ value \\
\hline \multicolumn{6}{|l|}{ Rice (n, \%) } \\
\hline Yes & $130(23.4)$ & $50(20.1)$ & $17(22.4)$ & $63(27.3)$ & 0.170 \\
\hline No & $426(76.6)$ & 199 (79.9) & $59(77.6)$ & $168(72.7)$ & \\
\hline \multicolumn{6}{|l|}{ Vegetables (n, \%) } \\
\hline Yes & $317(57.0)$ & $119(47.8)$ & $52(68.4)$ & $146(63.2)$ & $0.000 * *$ \\
\hline No & $239(43.0)$ & $130(52.2)$ & $24(31.6)$ & $85(36.8)$ & \\
\hline \multicolumn{6}{|l|}{ Fruit (n, \%) } \\
\hline Yes & $199(35.8)$ & 81 (32.5) & $29(38.2)$ & $89(38.5)$ & 0.352 \\
\hline No & $357(64.2)$ & $168(67.5)$ & $47(61.8)$ & $142(6 \mid .5)$ & \\
\hline \multicolumn{6}{|l|}{ Meat (n, \%) } \\
\hline Yes & $359(64.6)$ & $166(66.7)$ & $40(52.6)$ & $153(66.2)$ & 0.064 \\
\hline No & $197(35.4)$ & $83(33.3)$ & $36(47.4)$ & $78(33.8)$ & \\
\hline \multicolumn{6}{|l|}{ Fish (n, \%) } \\
\hline Yes & $228(4 I .0)$ & $116(46.6)$ & $22(28.9)$ & $90(39.0)$ & $0.017^{*}$ \\
\hline No & $328(59.0)$ & $133(53.4)$ & 54 (7I.I) & $|4|(6 \mid .0)$ & \\
\hline \multicolumn{6}{|l|}{ Egg (n, \%) } \\
\hline Yes & $153(27.5)$ & $60(24.1)$ & $26(34.2)$ & $67(29.0)$ & 0.180 \\
\hline No & $403(72.5)$ & $189(75.9)$ & $50(65.8)$ & $164(71.0)$ & \\
\hline \multicolumn{6}{|l|}{ Milk (n, \%) } \\
\hline Yes & $193(34.7)$ & 91 (36.5) & $25(32.9)$ & 77 (33.3) & 0.710 \\
\hline No & $363(65.3)$ & $158(63.5)$ & $51(67.1)$ & $154(66.7)$ & \\
\hline \multicolumn{6}{|l|}{ Water (n, \%) } \\
\hline Yes & $298(53.6)$ & $123(49.4)$ & 34 (44.7) & $|4|(6 \mid .0)$ & $0.010^{*}$ \\
\hline No & $258(46.4)$ & $126(50.6)$ & $42(55.3)$ & $90(39.0)$ & \\
\hline
\end{tabular}

Note: ${ }^{*} p<0.05,{ }^{*} p<0.01$.

Program" has been gradually implemented nationwide among school-age children since 2000, which provided milk to primary and secondary school students during a class interval. ${ }^{29}$ Moreover, the Nutrition Improvement Programme for Rural Compulsory Education Students (NIPRCES) was launched by the General Office of the State Council in China in 2011 to improve nutritional status of rural students. ${ }^{30}$ Chongqing included 12 concentrated and contiguous poverty-stricken areas as pilot counties of NIPRCES by providing students with the nutritional school lunch, milk, eggs, etc. ${ }^{31}$ The 12 areas included the surveyed areas of this study, however, the low proportion of meeting the recommended intake of milk, eggs and fish was found in this study. The reason for the results could be the low socioeconomic level of the three areas of Chongqing in this survey, the implementation and promotion of these programs may be inadequate in these areas. ${ }^{32}$ Relevant government departments should pay attention to this problem and take corresponding measures to improve dietary quality of school lunch in primary school students in these areas. Rice is the staple food for Han, Tujia, and Miao ethnic groups. ${ }^{11}$ A previous study showed that the average daily intake per standard person of cereals among Chinese residents has dropped by 102.6 g over past 10 years. ${ }^{33}$ Also, the low proportion of meeting the recommended intake of rice was found in this study, which was similar to a previous study, reporting that the intake of staple food (including rice, noodles, and other cereal food) for primary school students in grades 1-2 was insufficient at school lunch. ${ }^{34}$ "Eating a variety of foods, mainly cereals" is the first core dietary recommendation in CDG 2016, also is an important feature of a balanced diet in Chinese residents. Related nutritional education in terms of improving the cereals intake should be emphasized. 


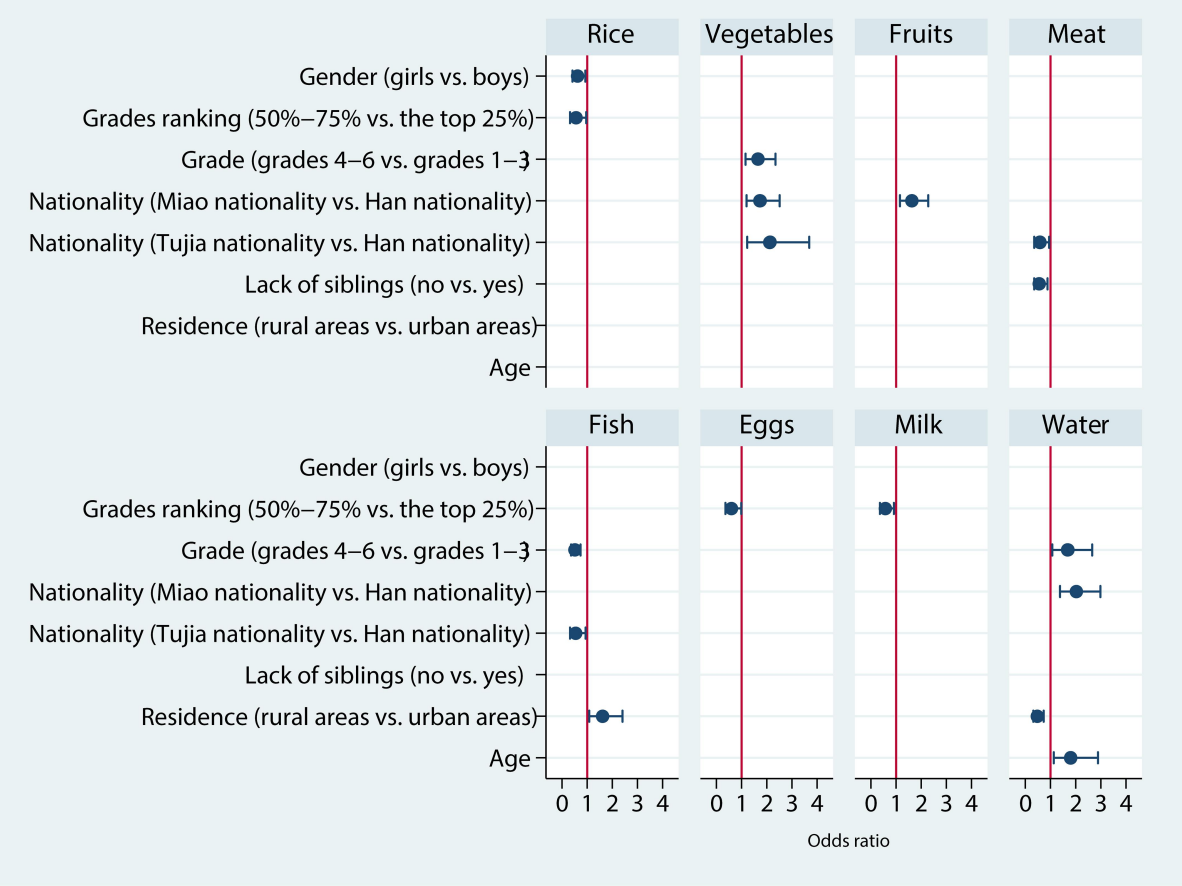

Figure I Stepwise logistic regression for identifying factors affecting dietary intake among primary school students in Chongqing, China.

In terms of eating habits, Tujia students had the highest proportion of usually having a light diet, and had the lowest proportion of usually eating sweet or fried foods among the three nationalities. This may reflect, at least partly, relatively better eating habits of Tujia nationality, which might be one reason for the lower prevalence of hypertension in Tujia nationality compared to Han nationality in Chongqing, China. ${ }^{35}$ Miao students had the lowest proportion of usually having a light diet. Special dietary preferences of Miao nationality may lead to the excessive intake of oil and salt. Miao population prefer to salted foods, such as salted vegetables, salted chicken, salted fish and salted meat, and fried glutinous rice ball and oiltea are their special snacks and drinks. ${ }^{36}$ In terms of dietary intake, Han students had the lowest proportion of meeting the recommended intake of vegetables, Tujia students had the lowest proportion of meeting the recommended intake of fish and water. Nutritional interventions should be considered to improve vegetables intake of Han students, and fish and water intake of Tujia students. The results of logistic regression analysis showed that comparing with Han nationality, Tujia students were more likely to meet the recommended intake of vegetables, while their meat and fish intake were less likely to meet the recommended intake than Han students. Besides, Miao students were more likely to meet the recommended intake of vegetables, fruits and water than Han students. Rice is the staple food for Miao and Tujia ethnic minorities, supplemented with miscellaneous grains (such as millet, sorghum, wheat and potatoes); additionally, Tujia nationality prefer soybean products, Miao nationality prefer glutinous rice and salted foods. ${ }^{11,35}$ Food patterns of Chinese residents are gradually switching as their income growth in recent years, with decreasing intake of traditional Chinese foods characterized by plant-based foods and increasing intake of animal-based foods. ${ }^{33}$ The speed of shift may be higher in Han nationality than that in ethnic minorities, as many ethnic minorities in China are socioeconomically disadvantaged relative to Han nationality. ${ }^{8}$ This is a possible explanation for the results of logistic regression. Previous studies reported a positive correlation between the energy contribution of animal sources to the diet and overweight/obesity whereas plant-based diets are either protective or no association with overweight/obesity in children and adolescents. ${ }^{37}$ Moreover, there is a certain degree of risk for plant-based diets in obtaining adequate nutrition to promote the normal growth and development of children and adolescents. ${ }^{38}$ Hence, the results may imply that some nutritional interventions should be conducted to prevent malnutrition among Tujia and Miao students and over-nutrition among Han students. Also, some factors, such as socioeconomic status, ways of 
preparing and consuming foods, traditional foods and customs of different ethnic groups should be considered during the nutritional interventions.

This study also revealed that gender, grades ranking, grade, the lack of siblings, residence and age were the influencing factors of rice, eggs, milk, vegetables, meat, fish and water intake. Therefore, these factors could be considered when designing nutritional interventions and implementing national dietary guidelines among primary school students. Compared with primary school students who had good grades ranking, students with poor grades ranking were less likely to meet the recommended intake of rice, eggs and milk. This was similar to a systematic review, which demonstrated that there was a positive association between overall diet quality and academic achievement. ${ }^{24}$ Schools or teachers should strengthen the opportunities to facilitate, support, and reinforce healthy dietary behaviours, especially for underachieving primary school students.

This study had certain limitations. Firstly, the use of cross-sectional survey data reduced the researchers' ability to make direct causal inferences. Longitudinal studies should be further conducted to confirm the findings of this study. Secondly, the sample size in the study was insufficient, especially the number of Tujia primary school students, which may result in less convincing results. Future studies need to expand the sample size of the three ethnic groups to make the comparison between different ethnic groups more convincing. Thirdly, primary school students may be too young to understand the questions of dietary intake. Though the investigators displayed and explained the food model of CDG 2016 on-site, and the method of one-onone interview-based questionnaire was used among primary school students in grades $1-2$, their inability to fully understand the questions of dietary intake is still a concern. The data of dietary intake obtained may not be accurate. It may be better to investigate their guardians or keep a record of dietary intake every day in further studies. Fourth, this questionnaire was designed by our research group, we invited experts in the field of dietary behaviour or student nutrition to modify the questionnaire. However, unvalidated measures in the questionnaire designed to measure the outcome variables may raise potential issues related to reliability. Finally, the study relied on self-report, which may introduce bias.

\section{Conclusion}

The low proportion of health-protective dietary behaviours among primary school students from Han, Tujia and Miao ethnic groups was found in this study. And there were differences in dietary behaviours among the three ethnic groups. This study could provide a reference for health professionals and educationalists to implement national dietary guidelines and conduct dietary interventions in consideration of socioeconomic status, ways of preparing and consuming foods, traditional foods and customs of different ethnic groups.

\section{Funding}

This study was supported by Chongqing science and technology communication and popularization project (cstc2016kp-sfhdB0002). The authors would like to thank all team members for their support and contributions to this study sincerely. And the authors would like to express their gratitude to all primary school students who agreed to participate in this survey.

\section{Disclosure}

The authors report no conflicts of interest in this work.

\section{References}

1. Shepherd J, Harden A, Rees R, et al. Young people and healthy eating: a systematic review of research on barriers and facilitators. Health Educ Res. 2006;21(2):239-257. doi:10.1093/her/cyh060

2. Scaglioni S, De Cosmi V, Ciappolino V, Parazzini F, Brambilla P, Agostoni C. Factors influencing children's eating behaviours. Nutrients. 2018;10(6):706. doi:10.3390/nu10060706

3. Montaño Z, Smith JD, Dishion TJ, Shaw DS, Wilson MN. Longitudinal relations between observed parenting behaviors and dietary quality of meals from ages 2 to 5. Appetite. 2015;87:324-329. doi:10.1016/j.appet.2014.12.219

4. Jones M, Pitt H, Oxford L, Bray I, Kimberlee R, Orme J. Association between food for life, a whole setting healthy and sustainable food programme, and primary school children's consumption of fruit and vegetables: a cross-sectional study in England. Int $J$ Environ Res Public Health. 2017;14(6):639. doi:10.3390/ijerph14060639

5. Census Office under the State Council of the People's Republic of China. Department of population and employment statistics of the national bureau of statistics of China. Tabulation on the 2010 population census of the people's republic of China. Beijing: China Statistics Press; 2012. Available from: http://www.stats.gov.cn/tjsj/pcsj/rkpc/6rp/ indexch.htm. Accessed April 29, 2020.

6. Hui-Fang L, Cai L, Wang XM, Golden AR. Ethnic disparities in prevalence and clustering of cardiovascular disease risk factors in rural Southwest China. BMC Cardiovasc Disord. 2019;19(1):200. doi:10.1186/s12872-019-1185-1

7. Liu X, Liu C, Schenck H, Yi X, Wang H, Shi X. The risk factors of 9-year follow-up on hypertension in middle-aged people in Tujia-Nationality settlement of China. J Hum Hypertens. 2017;31 (12):838-842. doi:10.1038/jhh.2017.58

8. Dong Y, Zou Z, Yang Z, et al. Prevalence of excess body weight and underweight among 26 Chinese ethnic minority children and adolescents in 2014: a cross-sectional observational study. BMC Public Health. 2018;18(1):562. doi:10.1186/s12889-018-5352-6 
9. Chen Z, Shu XO, Yang G, et al. Nutrient intake among Chinese women living in Shanghai, China. Br J Nutr. 2006;96(2):393-399. doi:10.1079/BJN20061829

10. WHO. Prioritizing areas for action in the field of population-based prevention of childhood obesity; 2012. Available from: https://www.who.int/ dietphysicalactivity/childhood/tools/en/. Accessed August 19, 2019.

11. Zhai F, He Y, Wang Z, Hu Y.[Status and characteristic of dietary intake of 12 minority nationalities in China]. J Hygiene Res. 2007;36 (5):539-541. Chinese.

12. Xu T, Liu J, Zhu G, Liu J, Han S. Prevalence of prehypertension and associated risk factors among Chinese adults from a large-scale multi-ethnic population survey. BMC Public Health. 2016;16 (1):775. doi:10.1186/s12889-016-3411-4

13. Sun Y, Cao Q, Zhang R.[Health- related behaviors in children in Han and minority populations in China]. Chinese General Practice. 2014;17(1):64-66.Chinese.

14. Suggs LS, Bella SD, Marques-Vidal P. Low adherence of Swiss children to national dietary guidelines. Prev Med Rep. 2016;3:244-249. doi:10.1016/j.pmedr.2016.03.004

15. U.S. Department of Health and Human Services and U.S. Department of agriculture. 2015-2020 dietary guidelines for Americans; 2015. Available from: http:/health.gov/dietaryguidelines/2015/guidelines/. Accessed December 30, 2019.

16. Chinese Nutrition Society. Chinese dietary guideline 2016. Beijing: People's Medical Publishing House; 2016. Available from: http://dg. cnsoc.org/article/04/8a2389fd5520b4f30155a5d93f1721c6.html. Accessed August 3, 2019.

17. World Health Organization Regional Office for South-East Asia. Regional consultation on food-based dietary guidelines for countries in asia region; 2012. Available from: https://apps.who.int/iris/handle/ 10665/205756. Accessed April 10, 2020.

18. Duan R, Liu Y, Xue H, Yang M, Cheng G.[Cross-sectional association between overall diet quality and overweight/obesity among children and adolescents in Chengdu]. Chin J Epidemiol. 2014;35(9):994. Chinese.

19. Shang X, Li Y, Liu A, et al. Dietary pattern and its association with the prevalence of obesity and related cardiometabolic risk factors among Chinese children. PLoS One. 2012;7(8):e43183. doi:10.1371/journal. pone. 0043183

20. Banfield EC, Liu Y, Davis JS, Chang S, Frazier-Wood AC. Poor adherence to US dietary guidelines for children and adolescents in the national health and nutrition examination survey population. $J$ Acad Nutr Diet. 2016;116(1):21-27. doi:10.1016/j.jand.2015.08.010

21. Mian L, Su F.[Current situation of dietary behaviors and nutrients intake in primary and secondary school students in southern part of Dongcheng District of Beijing in 2013]. Occup and Health. 2015;31 (11):1520-1522. Chinese.

22. Li H, Guan J, Ye H, Yang H. A survey of rural residents' perception and response to health risks from hot weather in ethnic minority areas in southwest China. Int J Environ Res Public Health. 2019;16 (12):2190. doi:10.3390/ijerph16122190

23. Asakura K, Todoriki H, Sasaki S. Relationship between nutrition knowledge and dietary intake among primary school children in Japan: combined effect of children's and their guardians' knowledge. J Epidemiol. 2017;27(10):483-491. doi:10.1016/j.je.2016.09.014

\section{Risk Management and Healthcare Policy}

\section{Publish your work in this journal}

Risk Management and Healthcare Policy is an international, peerreviewed, open access journal focusing on all aspects of public health, policy, and preventative measures to promote good health and improve morbidity and mortality in the population. The journal welcomes submitted papers covering original research, basic science, clinical \& epidemiological studies, reviews and evaluations,
24. Burrows T, Goldman S, Pursey K, Lim R. Is there an association between dietary intake and academic achievement: a systematic review. J Hum Nutr Diet. 2017;30(2):117-140. doi:10.1111/jhn.12407

25. Bleiweiss-Sande R, Chui K, Wright C, Amin S, Anzman-Frasca S, Sacheck JM. Associations between food group intake, cognition, and academic achievement in elementary schoolchildren. Nutrients. 2019;11(11):2722. doi:10.3390/nu11112722

26. Xu X, Liu D, Sharma M, Zhao Y. Prevalence and determinants of current smoking and intention to smoke among secondary school students: a cross-sectional survey among Han and Tujia nationalities in China. Int $J$ Environ Res Public Health. 2017;14(11):1323. doi:10.3390/ijerph14111323

27. Cahill LE, An P, Chiuve SE, et al. Fried-food consumption and risk of type 2 diabetes and coronary artery disease: a prospective study in 2 cohorts of US women and men. Am J Clin Nutr. 2014;100 (2):667-675. doi:10.3945/ajcn.114.084129

28. Turel O. Are energy drinks scapegoats? Decomposing teenagers' caffeine intake from energy drinks and soda beverages. Subst Use Misuse. 2018;53(12):2089-2092. doi:10.1080/10826084.2018.1441313

29. Ministry of agriculture and rural affairs of the People's Republic of China. Ministry of Agriculture: "School Milk Program" benefited nearly 5 million primary and secondary school students; 2010. Available from: http://www.moa.gov.cn/xw/zwdt/201009/ t20100919_1659146.htm. Accessed April 29, 2020.

30. Zhang F, Hu X, Tian Z, Zhang Q, Ma G. Literature research of the nutrition improvement programme for rural compulsory education students in China. Public Health Nutr. 2015;18(5):936-943. doi:10.1017/S1368980014001001

31. Chongqing Municipal Education Commission. Notification of the implementation of the nutrition improvement programme; 2016. Available from: http://jw.cq.gov.cn/zwgk_209/fdzdgknr/zcwj/qtwj/ 202003/t20200330_6729909.html. Accessed April 10, 2020.

32. Pu Y, Zhang F, Wang H, Hu M, Deng D.[Systematic review of the implementation of school milk program in China and its impact on growth and development in school-age children]. J China Med University. 2015;44(11):978-986. Chinese.

33. Zhao L, Fang Y, He Y, et al. [Trends of food consumption among Chinese population in 1992-2012]. Chinese General Practice. 2016;45(4):522-526. Chinese.

34. Huang Z, Gao R, Bawuerjiang N, Zhang Y, Huang X, Cai M. Food and nutrients intake in the school lunch program among school children in Shanghai, China. Nutrients. 2017;9(6):582. doi: 10.3390/nu9060582.

35. Zhou Y, Shen P, Fan M, Long W, Ge L.[Study on the relationship between dietary habits and hypertension in Tujia residents of Chongqing region]. Chongqing Med. 2016;45(29):4106-4109. Chinese.

36. Shang W, Zhu X.[A preliminary study of the diet culture among Miao nationality]. J Med Pharm Chinese Minorities. 2016;11(11):75-76. Chinese.

37. Sabate J, Wien M. Vegetarian diets and childhood obesity prevention. Am J Clin Nutr. 2010;91(5):1525s1529s. doi:10.3945/ajen.2010.28701F

38. Newby PK. Plant foods and plant-based diets: protective against childhood obesity? Am J Clin Nutr. 2009;89(5):1572s1587s. doi:10.3945/ajen.2009.26736G

guidelines, expert opinion and commentary, case reports and extended reports. The manuscript management system is completely online and includes a very quick and fair peer-review system, which is all easy to use. Visit http://www.dovepress.com/testimonials.php to read real quotes from published authors. 